\title{
Predicting positive surgical margins in partial nephrectomy: A prospective multicentre observational study (the RECORd 2 project)
}

\author{
Riccardo Schiavina ${ }^{\mathrm{a}}$, Andrea Mari ${ }^{\mathrm{b}}$, Lorenzo Bianchi ${ }^{\mathrm{a}}$, Daniele Amparore ${ }^{\mathrm{c}}$, \\ Alessandro Antonelli ${ }^{\mathrm{d}, \mathrm{e}}$, Walter Artibani ${ }^{\mathrm{e}}$, Eugenio Brunocilla ${ }^{\mathrm{a}}$, Umberto Capitanio ${ }^{\mathrm{f}}$, \\ Cristian Fiori ${ }^{\mathrm{c}}$, Fabrizio Di Maida ${ }^{\mathrm{b}}$, Paolo Gontero ${ }^{\mathrm{g}}$, Alessandro Larcher ${ }^{\mathrm{f}}$, \\ Vincenzo Li Marzi ${ }^{\mathrm{h}}$, Nicola Longo ${ }^{\mathrm{i}}$, Giancarlo Marra ${ }^{\mathrm{g}}$, Emanuele Montanari ${ }^{\mathrm{j}}$, \\ Francesco Porpiglia ${ }^{\mathrm{c}}$, Marco Roscigno ${ }^{\mathrm{k}}$, Claudio Simeone ${ }^{\mathrm{d}}$, Salvatore Siracusano ${ }^{\mathrm{e}}$, \\ Riccardo Tellini ${ }^{\mathrm{b}}$, Carlo Terrone ${ }^{1}$, Donata Villari ${ }^{\text {h}}$, Vincenzo Ficarra ${ }^{\mathrm{m}}$, Marco Carini ${ }^{\mathrm{b}}$, \\ Andrea Minervini ${ }^{\text {b, }}$, Collaborators \\ a Department of Urology, University of Bologna, Department of Experimental, Diagnostic, and Specialty Medicine, University of Bologna, Bologna, Italy \\ ${ }^{\mathrm{b}}$ Department of Urology, University of Florence, Unit of Oncologic Minimally-Invasive Urology and Andrology, Careggi Hospital, Florence, Italy \\ ${ }^{c}$ Division of Urology, Department of Oncology, San Luigi Gonzaga Hospital, School of Medicine, Orbassano, Turin, Italy \\ d Department of Urology, Spedali Civili Hospital, University of Brescia, Brescia, Italy \\ e Department of Urology, Azienda Ospedaliera Universitaria Integrata (A.O.U.I.), Verona, Italy \\ ${ }^{\mathrm{f}}$ Unit of Urology, Division of Experimental Oncology, URI-Urological Research Institute, Vita-Salute San Raffaele University, IRCCS San Raffaele Scientific \\ Institute, Milan, Italy \\ ${ }^{g}$ Division of Urology, Department of Surgical Sciences, San Giovanni Battista Hospital, University of Studies of Torino, Turin, Italy \\ h Department of Urology, University of Florence, Unit of Urological Minimally Invasive Robotic Surgery and Renal Transplantation, Careggi Hospital, \\ Florence, Italy \\ ${ }^{\mathrm{i}}$ Department of Urology, University Federico II of Naples, Naples, Italy \\ ${ }^{j}$ Department of Urology, Fondazione IRCCS Ca' Granda, Ospedale Maggiore Policlinico, Policlinico, University of Milan, Milan, Italy \\ ${ }^{\mathrm{k}}$ Department of Urology, Papa Giovanni XXIII Hospital, Bergamo, Italy \\ ${ }^{1}$ Department of Urology, Policlinico San Martino Hospital, University of Genova, Italy \\ m Department of Human and Paediatric Pathology Gaetano Barresi, Urologic Section, University of Messina, Messina, Italy
}

\section{A R T I C L E I N F O}

\section{Article history:}

Received 11 December 2019

Accepted 15 January 2020

Available online $\mathrm{xxx}$

\section{Keywords:}

Surgical margins

Nephron-sparing surgery

Nomogram

Partial nephrectomy

Renal cell carcinoma

Robot-assisted partial nephrectomy

\begin{abstract}
A B S T R A C T
Purpose: to evaluate clinical predictors of positive surgical margins (PSMs) in a large multicenter prospective observational study and to develop a clinic nomogram to predict the likelihood of PSMs after partial nephrectomy (PN).

Materials and methods: We prospectively evaluated 4308 patients who had surgical treatment for renal tumors between January 2013 and December 2016 at 26 urological Italian Centers (RECORd 2 project). Two multivariable logistic models were evaluated to predict the likelihood of PSMs. Center caseload was dichotomized using a visual assessment adjusted for several predictors of PSMs. A nomogram predicting PSMs was developed.

Results: Overall, 2076 patients treated with PN were evaluated. pT1a, pT1b, pT2 and pT3a were recorded in $68.7 \%, 22.6 \%, 2.1 \%$ and $6.6 \%$ of the patients, respectively. PSMs were recorded in $342(16.5 \%)$ patients. From a null multivariable model against number of PN/year, $60 \mathrm{PN} /$ year were identified as the best cutoff to define a high-volume centre. At multivariable analysis, clinical stage (cT1a vs. cT2 [OR 1.94]; $\mathrm{p}=0.03$ ), volume centre ( $\leq 60 \mathrm{PN} / \mathrm{year}$ ) (OR 2.22; $\mathrm{p}<0.0001$ ), imperative vs elective indication (OR 2.10; $\mathrm{p}=0.04$ ), surgical technique (laparoscopic vs. open [OR 1.62; $\mathrm{p}=0.002$ ), lymphovascular invasion (OR 2.27; $\mathrm{p}=0.01)$ and upstaging to PT3a (OR 2.81; $<<0.0001)$ were independent predictors of PSMs. The final nomogram included age, ASA score, Charlson score, clinical tumor stage, surgical indication, surgical approach, surgical technique, PADUA score, clamp procedure and volume centre.
\end{abstract}

\footnotetext{
* Corresponding author. Department of Urology, University of Florence, Careggi Hospital, San Luca Nuovo, Firenze, Italy.

E-mail address: andrea.minervini@unifi.it (A. Minervini).
} 
Conclusions: PSMs after PN were significantly more likely in patients with lower clinical stage, higher PADUA score, in individuals referred to laparoscopic PN and in those treated at lower volume centers. We used these data to develop a nomogram to predict such risk.

(C) 2020 Elsevier Ltd, BASO The Association for Cancer Surgery, and the European Society of Surgical Oncology. All rights reserved.

\section{Introduction}

Current international guidelines [1,2] state that partial nephrectomy (PN) is the treatment of choice for clinical T1 RCC regardless of the surgical approach. Despite the functional benefits offered by conservative surgery and comparable oncologic outcomes compared to radical nephrectomy [3], the potential risk of positive surgical margins (PSMs) remains a matter of concern [4]. Indeed, the incidence of PSMs after PN ranges variously from $0 \%$ to $7 \%$ [5] with an increasing risk for smaller, high-grade tumors or in imperative cases, regardless of the surgical technique [6]. Although the clinical role of PSMs on cancer recurrence and mortality is still controversial, avoiding PSMs is considered crucial in PN. Of note, local recurrence seems to be more likely in patients with PSMs $[6,7]$, especially in high risk tumors that by themselves may have a stronger risk of recurrence $[4,8,9]]$. Contrarily, most studies found no significant impact on cancer-specific survival by PSM $[4,10,11]$. However, considering the long natural history of T1 RCC undergone surgical management, overall survival (OS) should also be regarded as one of the more relevant quality control measures after PN [12]. As consequence, some evidences [12,13] suggest that PSMs patients are at a higher risk of shorter overall survival, despite a possible confounding effects from age and comorbidity. Currently, clinical and surgical models aiming to predict the occurrence of PSMs after PN are lacking in literature. The aims of the present study are: 1) to identify the predictors of PSMs after PN basing on a rigorous prospectively recorded web-based database from a national collaborative project, and 2) to develop a clinic nomogram to predict the likelihood of PSMs after PN.

\section{Materials and methods}

\section{Study population}

The Italian REgistry of COnservative and Radical Surgery for cortical renal tumour Disease (RECORD 2 Project) is a prospective observational multicentre project promoted by the Italian Society of Urology (SIU). This study was approved by the local ethics committee and informed consent was collected for all the patients. Overall 4325 consecutive patients who underwent renal surgery for cortical renal tumors at 26 urological Italian centers between January 1st, 2013 and December 31st, 2016 were included. Of these, 1712 patients treated with radical nephrectomy, 29 patients with missing data and 508 patients treated with PN and with benign histotype were excluded, leading a final population of 2076 individuals.

An online and centrally controlled data server was generated to limit missing or wrong data inputs. All data of patients undergoing surgery were prospectively recorded by medical doctors. The database included 6 main folders: 1) anthropometric and preoperative data; 2 ) imaging, indications (elective, relative and absolute) and co-morbidities; 3 ) intra-operative data; 4) post-operative data; 5) histological analysis 6 ) follow-up.

\section{Covariates}

Comorbidity status was evaluated by Charlson comorbidity index
(CCI), physical status by the American Society of Anesthesiologists (ASA) classification system. Surgical indications were defined as elective (unilateral lesion with healthy contralateral kidney), relative (presence of diabetes, hypertension or lithiasis that could potentially affect kidney function in the future) and absolute (bilateral tumors, multiple tumors, moderate to severe CKD or tumors involving an anatomically or functionally solitary kidney). The Preoperative Aspects and Dimensions Used for an Anatomical (PADUA) score was calculated to assess the nephrometric complexity of each case [14]. Centre caseload was defined as the number of PNs per year in each centre.

\section{Pathological evaluation}

All surgical specimens were processed according to standard pathological procedures at each institution by experienced uropathologists. For surgical margins evaluation the specimens were fixed in $10 \%$ buffered formalin, and grossly analyzed. The size, the colour, the gross aspect (solid to cystic) were recorded and the surgical margin was marked with ink. After tumor dissection, samplings were performed in order to obtain tissue blocks where tumor, renal parenchyma, and surgical edges were comprised and further blocks where tumor, renal capsule, and peritumoral fat were enclosed. The margin was considered positive when tumor tissue was marked with ink. The margin was considered negative when non-neoplastic renal tissue was observed between tumor tissue and the ink line.

The grading system adopted by the International Society of Urological Pathology (ISUP) illustrated in the fourth edition of the WHO classification tumors of the urinary system and male genital organs (2016) was used. No central pathological slide review was performed.

\section{Outcome}

Primary outcome of the study was to assess the incidence and the predictors of PSMs after PN, basing on a rigorous prospectively recorded web-based database from a national collaborative project.

\section{Statistical analysis}

First, clinical, pathological, intraoperative and perioperative characteristics of the study population were assessed. Second, a univariate and a clinical model-based multivariable logistic regression to predict PSMs was performed, including the following co-variates: age (continuous variable), ASA score (continuous variable), CCI score (continuous variable), clinical tumor stage (cT1a vs. cT2 and cT1b vs. cT2), surgical indication (relative vs. elective and imperative vs. elective), surgical approach (open vs. laparoscopic vs. robotic), surgical technique (enucleoresection vs simple enucleation) PADUA score (continuous variable), clamp procedure (yes vs. no) and centre volume (PN/year). Moreover, a clinico-pathological model-based multivariable logistic regression to predict PSMs was performed, adding to clinical also pathological co-variates such as lymphovascular invasion (yes vs. no), upstaging to pT3a (yes vs. no) and nucleolar grading (3-4 vs. 1-2). The area under the receiving operator characteristic (ROC) curves (AUC) were used to 
quantify the predictive discrimination. Different ROC curves using the same set of observations were compared using the roccomp function. Third, regression-based coefficients of the clinical modelbased multivariable logistic regression, were used to develop a nomogram predicting the likelihood of PSMs. Fourth, decision curve analysis was applied to determine whether the clinical value of the newly derived model increased the net benefit over a realistic range of threshold probabilities. Finally, center caseload was dichotomized using a visual assessment of the functional form of the association of center caseload with PSMs. In detail, a plot of Martingale residuals from the null multivariable clinical model against center caseload was set. Statistical significance was defined as $\mathrm{p}<0.05$. All tests were two-sided. Analyses were carried out using STATA v.14.1 (StataCorp LP, College Station, TX).

\section{Results}

\section{Clinical, surgical features and complications}

The preoperative clinical characteristics of patients included in the study are summarized in Table 1 . The median (interquartile range [IQR]) age was 62 (54-72) years. The median (IQR) ASA and CCI score were $2(2-3)$ and $1(0-2)$, respectively. Overall, 1485 (71.5\%), 539 (26.0\%) and $52(2.5 \%)$ lesions were classified as clinical T1a, T1b and T2 stage, respectively. The median (IQR) PADUA score was 8 (7-9) and a PADUA score $\geq 10$ was assessed in $16.2 \%$ of the patients. The surgical indication to PN resulted elective, relative and imperative in $83.9 \%, 12.5 \%$ and $3.6 \%$ of the patients, respectively.

The perioperative surgical features and complications are shown in Table 2. Patients were treated with open, laparoscopic and robot-assisted PN in 36\%, 27,6\% and 36,4\% of cases, respectively. The surgical procedure was recorded as off-clamp in $45,8 \%$ and onclamp in $54.2 \%$ of cases with median warm ischemia time (WIT) of $16 \mathrm{~min}$. Overall, 722 (34.8\%) cases were simple enucleation and 1354 (65.2\%) were standard PN. Median (IQR) centre volume was 66 (41-84) PNs/year with $72.9 \%$ of the patients treated in centers with volume $>50 \mathrm{PN} /$ year. Overall, intraoperative complication rate was 3.8\%. Medical and surgical postoperative complications were $3,6 \%$ and $10,8 \%$, respectively.

\section{Pathological features}

Pathological characteristics of the study population are depicted in Table 3. A pathological stage T1a (pT1a), pT1b, pT2 and pT3a was assessed in $68.7 \%, 22.6 \%, 2.1 \%$ and $6.6 \%$ of the patients, respectively, with pathological nodal involvement (pN1) in $0.4 \%$ of cases. A PSM was found at final pathology in 342 (16.5\%) individuals. Lymphovascular invasion, urinary calyceal system involvement, perinephric fat infiltration, tumor necrosis and sarcomatoid differentiation were reported in $4.6 \%, 1.8 \%, 1.1 \%, 16.5 \%$ and $1.1 \%$ of the pathological specimens, respectively.

\section{Multivariable models predicting positive surgical margins}

The results of multivariable logistic regression models are depicted in Table 4. The clinical-based multivariable model revealed that clinical stage (namely, cT1a vs. cT2 [OR 2.17, 95\% CI 1.04-3.22], $\mathrm{p}=0.002$ ), PADUA score (OR 1.12, 95\% CI 1.00-1.26; $\mathrm{p}=0.0045$ ), volume centre (namely, $\leq 60 \mathrm{PN} /$ years [OR $2.22,95 \% \mathrm{CI}$ $1.56-3-14 ; \mathrm{p}<0.0001$ ) and surgical technique (namely, laparoscopic vs. open [OR 1.52, 95\% CI 1.01-2.27]; $\mathrm{p}=0.03$ ) were independent predictors of PSMs (AUC: 0.66). The clinico-pathological multivariable model revealed that clinical stage (namely, cT1a vs. cT2 [OR 1.94, 95\% CI 1.03-2.79]; $\mathrm{p}=0.03)$, volume centre ( $\leq 60 \mathrm{PN} /$ year) (OR 2.22, 95\% CI 1.55-3.18; $\mathrm{p}<0.0001$ ), imperative indication vs elective indication (OR 2.10 95\% CI 1.01-4.37), surgical technique (namely, laparoscopic vs. open [OR 1.62, 95\% CI 1.07-2.43]; $\mathrm{p}=0.002$ ), lymphovascular invasion (OR 2.27, 95\% CI 1.19-4.17; $\mathrm{p}=0.01$ ) and upstaging to pT3a (OR 2.81, 95\% CI 1.72-4.59; $\mathrm{p}<0.0001$ ) were independent predictors of PSMs (AUC: 0.70 ).

\section{Nomogram predicting positive surgical margins}

The coefficients of covariates included in the clinical model-base multivariate logistic regression (namely, age, ASA score, CCI score, clinical tumor stage, surgical indication, surgical approach, surgical technique, PADUA score, clamp procedure and volume centre) were used to develop a novel nomogram aimed to predict the occurrence of PSMs after PN (Fig. 1). The ROC AUC of the model was 66\%. This model showed a significantly higher predictability compared to each unmodifiable tumor characteristics, such as clinical tumor stage (AUC ROC: $51.9 \%, \mathrm{p}<0.0001$ ), PADUA score (AUC ROC: $52.4 \%$, $\mathrm{p}=0.0001)$. At the decision curve analysis, the nomogram revealed clinical net benefit with a threshold probability of $\geq 4 \%$ (Fig. 2). At plotting Martingale residuals from a null multivariable model against number of PN/year (as surrogate as centre volume), we identified the best cut-off to define a high-volume centre as $60 \mathrm{PN} /$ year (Fig. 3).

\section{Discussion}

A complete tumor removal is an oncologic principle of paramount importance that impacts on the surgical success of PN. Despite the lack of uniform agreement in clinical experience, local recurrence seems to be more likely in patients with PSMs [15]. It is important to note that data from multiple studies have suggested that patients with PSM are at risk for recurrence in case of high risk tumors defined by features that by themselves may have a stronger risk of relapse (namely, increasing tumor size, pT3a stage and higher grade) $[4,8,9]$. On the contrary, in low-grade primary tumors a low malignant potential of PSMs is suggested due to low cancer progression rate. Additionally, destruction of tumor cells due to coagulation, mechanical stress, or induced ischemic insult during nephron-sparing surgery (NSS) may limit the survival and propagation of malignant cells at the resection boundary [9]. As consequence, most studies found no impact on cancer-specific survival by PSM $[4,10]$. However, due to the slow growth rate, most studies are limited to assess the impact of PSM on long term mortality due to the inadequate follow-up and for not considering the competing effect of several confounders such age and comorbidity [12]. Therefore, the identification of predictors of PSM at PN is crucial and a predictive model for PSM after PN actually lacks in literature. Several aspects of our work are noteworthy. First, the overall PSMs rate in a high-volume multicentric cohort was $16.5 \%$. This finding is higher compared to previous data reported in literature [5], however $68.7 \%$ of cases were pT1a, roughly $20 \%$ had high-grade tumor and $16.1 \%$ of patients underwent PN with non-elective indication: previous authors reported that low stage, high grade tumor and imperative indication to PN increase the risk of PSMs [4]. Second, at clinical-based multivariable model, age at surgery (OR:1.02), clinical stage (cT1a vs cT2; OR: 2.17), PADUA score (OR: 1.12) center volume ( $\leq 60 \mathrm{PN} / \mathrm{year}$; OR: 2.22) and laparoscopic vs. open approach (OR: 1.52) emerged as independent predictors of PSMs after PN. Aged patients referred to NSS had several conditions both tumor-related and patients-related that might suggest imperative indication to PN, increasing the risk of PSM. Moreover, older individuals with complex renal masses unsuitable for percutaneous ablation could have higher risk of PSM in case of PN [16]. According to previous data, T1a RCC presents higher risk of PSMs since small tumor size might prevent the surgeon from accurately estimate 
Table 1

Preoperative characteristics of 2076 patients treated with partial nephrectomy for renal cell carcinoma (RCC).

\begin{tabular}{|c|c|c|c|}
\hline \multicolumn{4}{|c|}{ Preoperative characteristics $(\mathrm{n}=2076)$} \\
\hline \multirow[t]{2}{*}{ Gender } & - Male & 1443 & $69.5 \%$ \\
\hline & - Female & 633 & $30.5 \%$ \\
\hline \multicolumn{2}{|l|}{ Age (years) } & 62.6 & $54.7-72.0$ \\
\hline \multicolumn{2}{|l|}{ BMI $(\mathrm{kg} / \mathrm{m} \prec 2)$} & 26.9 & $23.9-28.8$ \\
\hline \multirow[t]{2}{*}{ ECOG Score } & median IQR & 0 & $0-1$ \\
\hline & - $\geq 1$ & 618 & $29,8 \%$ \\
\hline \multirow[t]{2}{*}{ ASA PS Score } & median IQR & 2 & $2-3$ \\
\hline & - $\geq 3$ & 572 & $27.6 \%$ \\
\hline \multicolumn{2}{|l|}{ CCI PS score } & 1 & $0-2$ \\
\hline \multicolumn{2}{|l|}{ AA-CCI PS score } & 4 & $2-5$ \\
\hline \multirow[t]{3}{*}{ Surgical indication } & - Elective & 1724 & $83.9 \%$ \\
\hline & - Relative & 257 & $12.5 \%$ \\
\hline & - Imperative & 74 & $3.6 \%$ \\
\hline \multirow[t]{2}{*}{ Tumor side. n. \% } & - Right & 1066 & $51.4 \%$ \\
\hline & - Left & 1010 & $48.6 \%$ \\
\hline \multicolumn{4}{|l|}{ Clinical T, n. \% } \\
\hline & - T1a & 1485 & $71.5 \%$ \\
\hline & - $\mathrm{T} 1 \mathrm{~b}$ & 539 & $26.0 \%$ \\
\hline & $\bullet \mathrm{T} 2$ & 52 & $2.5 \%$ \\
\hline \multicolumn{4}{|c|}{ Tumor growth pattern } \\
\hline & - $\geq 50 \%$ Exophytic & 1165 & $56.1 \%$ \\
\hline & - $<50 \%$ Exophytic & 740 & $35.6 \%$ \\
\hline & - Entirely endophytic & 171 & $8.3 \%$ \\
\hline \multicolumn{4}{|c|}{ Tumor location relative to the polar line (PL) } \\
\hline & - Entirely above PL & 1095 & $52.7 \%$ \\
\hline & - $\leq 50 \%$ crosses PL & 701 & $33.9 \%$ \\
\hline & - $>50 \%$ crosses PL & 280 & $13.4 \%$ \\
\hline \multicolumn{4}{|c|}{ Nearingness to the collecting system } \\
\hline & - $\geq 7$ & 1142 & $55.0 \%$ \\
\hline & - $>4$ but $<7$ & 367 & $17.7 \%$ \\
\hline & $\bullet \leq 4$ & 567 & $27.3 \%$ \\
\hline \multicolumn{2}{|c|}{ PADUA score, median IQR } & 8.0 & $7.0-9.0$ \\
\hline \multicolumn{4}{|c|}{ PADUA score complexity index } \\
\hline & - $6-7$ & 1041 & $50,1 \%$ \\
\hline & - $8-9$ & 699 & $33.7 \%$ \\
\hline & $\bullet \geq 10$ & 336 & $16.2 \%$ \\
\hline \multicolumn{2}{|c|}{ Baseline hemoglobin (mg/dL), median (IQR) } & 14.3 & $13.3-15.2$ \\
\hline \multicolumn{2}{|c|}{ Baseline creatinine (mg/dL), median (IQR) } & 0.9 & $0.8-1.1$ \\
\hline \multicolumn{2}{|c|}{ Baseline eGFR ( $\mathrm{mL} / \mathrm{min})$, median IQR } & 84.6 & $69.5-100.4$ \\
\hline
\end{tabular}

tumor extension and thereby provoke a PSM [17]. A missing or incompletely developed pseudocapsule, particularly present in smaller tumors, may also contribute to higher PSM rates [18]. Finally, accidental disintegration of the resection margin, is more likely to occur in smaller tumors $[4,19,20]$. Despite PADUA score was developed to predict the surgical complexity of renal masses and the postoperative complications, individuals with higher PADUA score are at higher risk of PSMs due to tumor's complexity.

The effect of surgical approach on PSM is still debated. In experienced hands, the overall PSM seems to be comparable within open, laparoscopic and robotic approach [21,22]. However, in our multicentric cohort individuals treated with laparoscopic approach had higher risk of PSMs. Indeed, the higher surgical difficulties related to laparoscopic technique and the higher proportion of patients treated with clampless approach in LPN group, may justify that the margins of resection can be hidden by intraoperative bleeding resulting in higher PSM rates.

The center volume was found to be the strongest independent predictor of PSMs for both clinical and clinico-pathological model. Of note PSM, together with warm ischemia time and complications, represents the main outcome of the learning curve process in PN, that might be longer and more complex than expected [23]. Indeed, PSMs are more related to surgeon-related factors (effect of caseload and experience) [24] and volume center, rather than patient's and tumor related factors. Therefore, the center caseload, that reflects the experience of both surgeons and surgical team might have a
Table 2

Operatory characteristics of 2076 patients treated with partial nephrectomy for renal cell carcinoma (RCC).

\begin{tabular}{lll}
\hline Perioperative characteristics $(\mathrm{n}=2076)$ & & \\
\hline Surgical approach, n. \% & & \\
- Open & 748 & $36.0 \%$ \\
- Laparoscopic & 572 & $27.6 \%$ \\
- Robotic & 756 & $36.4 \%$ \\
Type of resection, n. \% & & \\
- Enucleation & 722 & $34.8 \%$ \\
- Standard PN & 1354 & $65.2 \%$ \\
Pedicle clamping, n. \% & & \\
- Off-clamp & 950 & $45.8 \%$ \\
- On-clamp & 1126 & $54.2 \%$ \\
- Warm ischemia time, median IQR & 16 & $12-20$ \\
Volume centre (PN/year in each centre), median IQR & 66 & $41-84$ \\
Patients treated in centers performing $>60$ PN/year & 1140 & $54.9 \%$ \\
Operatory time (minutes), median IQR & 137 & $105-180$ \\
Estimated blood loss (cc), median IQR & 150 & $80-295$ \\
Intraoperative complications, n. \% & 80 & $3.8 \%$ \\
Medical postoperative complications, n. \% & 74 & $3.6 \%$ \\
Surgical postoperative complications, n. \% & 225 & $10.8 \%$ \\
Transfusion rate, n. \% & 148 & $7.1 \%$ \\
\hline
\end{tabular}

greater impact on predicting the likelihood of PSMs rather than the surgical technique itself. Peyronnet et al. [25] stated that a caseload of $50 \mathrm{PN} /$ year significantly reduced PSMs, length of hospital stay, WIT and operative time, suggesting that PN should be performed and centralize in high volume center ( $>50$ PN/year). Accordingly, Couapel et al. [26] found that mild-low volume centers ( $<10$ PN in 6 months) experienced significantly higher risk of PSMs, mainly due to higher adoption of laparoscopic techniques rather than open or robotic approaches mainly used in high volume center. Similarly, we identified the number of PN/year as a surrogate of the volume center and we found that it was significantly associated with the occurrence of PSMs. After adjusting for several clinical patients' and tumors' characteristics, a threshold of $60 \mathrm{PN} /$ year was identified and it was inserted in both clinical and clinic-pathological models. Patients treated in centers performing $\leq 60$ PN/year were associated with a risk of PSMs more than double. A reasonable explanation might reside in several reasons: the surgeons and the surgical team long experience, the higher use of the robotic platform in high-volume center, standardization of the surgical technique regardless surgical approach and maximization of peri-operative patients care.

We found that surgical technique (namely, simple enucleation vs. standard PN) was not related to higher risk of PSMs. Indeed, as previously reported, despite patients who underwent simple enucleation are exposed to the risk of a PSMs, since a pseudocapsule serving as landmark for dissection could be missing in some tumors, simple enucleation allows to achieve negative surgical margins in the vast majority of patients providing excellent midterm local control and oncologic outcomes [4,27]. Similarly, the renal hilum clamping approach was not found to reduce the risk of PSM compared to off clamp procedures [28,29]. Some authors proposed optimal hilar clamping to avoid bleeding that worsens the resection quality and exposes to the risk of PSM, in order to improve visualization of the resection margin. Indeed, higher proportion of PSMs after zero ischemia (5.6\%) compared to cold (4.8\%) or warm $(4.0 \%)$ ischemia suggests that the ischemia technique may influence the resection technique, resulting in higher PSMs and local recurrence rates. However, according to our findings, systematic reviews of the literature showed no impact of the technique used to manage the renal pedicle on either surgical or oncological outcomes of PN [30]. The correlation of lymphovascular invasion and pT3a stage with the incidence of PSM still remains 
Table 3

Pathological results in 2076 patients treated with partial nephrectomy for renal cell carcinoma (RCC)

\begin{tabular}{llll}
\hline Pathologic results $(\mathrm{n}=2076)$ & & & \\
\hline Histotype, $\mathrm{n} \%$ & Clear cell RC n, (\%) & 1461 & $70.4 \%$ \\
& Papillary RCC & 405 & $19.5 \%$ \\
& Chromophobe RCC & 191 & $9.2 \%$ \\
& Unclassified RCC & 2 & $0.1 \%$ \\
& Other renal tumors & 17 & $0.8 \%$ \\
Pathological T stage, n \% & pT1a & 1427 & $68.7 \%$ \\
& pT1b & 469 & $22.6 \%$ \\
& pT2 & 43 & $2.1 \%$ \\
& pT3a & 137 & $6.6 \%$ \\
Pathological N stage, n \% & pNx & 1752 & $84.4 \%$ \\
& pN0 & 263 & $12.7 \%$ \\
Nucleolar Grading & G1 & 8 & $0.4 \%$ \\
& G2 & 279 & $13.4 \%$ \\
& G3 & 1187 & $57.2 \%$ \\
G4 & 400 & $19,3 \%$ \\
Positive surgical margins, n \% & Not applicable & 33 & $1.6 \%$ \\
Lymphovascular invasion, n \% & & 176 & $8.5 \%$ \\
Urinary calyceal system invasion, n \% & 342 & $16.5 \%$ \\
Perinephric fat invasion, n \% & & 95 & $4.6 \%$ \\
Tumor necrosis, n \% & 38 & $1.8 \%$ \\
\% of tumor necrosis, median IQR & 22 & $1.1 \%$ \\
Sarcomatoid differentiation, n \% & 342 & $16.5 \%$ \\
\hline
\end{tabular}

controversial. Indeed, irregular shape and infiltrative growth pattern may be related to more aggressive biology of the disease and it may render the tumor dissection more challenging thus enhancing the incidence of PSM [20].

To our knowledge, this is the first available nomogram to predict the likelihood of PSMs after PN in a multicentric cohort. Despite suboptimal accuracy, this model showed a significantly higher predictability compared to each unmodifiable tumor characteristics, such as clinical tumor stage and PADUA score. Moreover, the decision curve analysis showed that this statistical model would improve the clinical decision-making when the predicted risk was $>4 \%$. Finally, our model could be useful to identify the modifiable predictors of PSMs in RCC patients scheduled for PN (i.e center caseload and surgical approach) to offer the highest chance to obtain optimal cancer control.

In this contest, we identified future perspectives to prevent PSMs: 1) the improvement of novel technologies applicated to robotic surgery such as the intraoperative ultrasound to define the tumor border and growth and the near-infrared fluorescence imaging can be valuable in correctly defining tumor extent, intended resection line and renal vasculature; 2 ) the utilization of 3D models and augmented reality can improve the knowledge of tumor's anatomy and vascularization allowing better intraoperative plan for ideal selective clamping: optimal ischemia in the area nearby the tumor with safe plane of dissection, potentially reducing PSM and better preserving the remnant health parenchyma by ischemic damage; 3 ) to accredit only high-volume canters to perform PN might contribute to implement the adoption of such novel technologies, thus reducing PSMs and maximizing outcomes and cost-effectiveness of PN.

Our study is not devoid from limitations. First, despite the highquality data collection was guaranteed by the rigorous control of data prospectively inserted on the Internet-based platform only by medical doctors from the participating centers, data were retrospectively analyzed, and they could be biased from inherent and not modifiable confounding factors. Second, our database lacks some fields as clinical tumor size, the use of intraoperative tools (i.e ultrasound, near-infrared fluorescence) and the surgical approach (namely, transperitoneal vs. retroperitoneal), whose impact on PSMs was not consequently explored. Third, due to its multicentric design, the surgical plan concerning type of resection (simple enucleation vs. standard resection), arterial clamping technique and surgical approach (open, laparoscopic or robotic) was left to the surgeons' preference. Moreover, the adoption of robotic technology was chosen according to the center availability and to the preference of the surgeons, with potential implication in free-margin achievement. Fourth, no central pathological revision was performed and the report of PSMs could be biased within centers. Fifth, in the present study we were not able to determine the experience and the learning curve of each surgeon, hence the center caseload (expressed by the number of PNs per year) was chosen as surrogate of center experience. Finally, despite this nomogram was tested on the largest nation-based prospective database on PN available to date, our nomogram lacks an external validation. Thus, the validity of the proposed nomogram to predict PSMs should be tested on other external cohorts, in order to be generalizable to all patients undergoing PN.

Table 4

Multivariable logistic regression models to predict positive surgical margins (PSMs).

\begin{tabular}{|c|c|c|c|c|c|c|c|c|c|}
\hline \multirow[t]{3}{*}{ Variables } & \multirow{2}{*}{\multicolumn{3}{|c|}{ Univariable analysis }} & \multicolumn{6}{|c|}{ Multivariable analysis for positive SM } \\
\hline & & & & \multicolumn{3}{|c|}{ Clinical Model } & \multicolumn{3}{|c|}{ Clinico-pathological Model } \\
\hline & OR & CI $95 \%$ & P-value & OR & CI 95\% & P-value & OR & CI 95\% & P-value \\
\hline Age (continuous variable) & 1.02 & $1.00-1.03$ & 0.02 & 1.02 & $0.99-1.03$ & 0.047 & 1.01 & $0.99-1.02$ & 0.09 \\
\hline ASA score (continuous variable) & 1.37 & $1.06-1.75$ & 0.01 & 1.13 & $0.84-1.52$ & 0.42 & 1.11 & $0.82-1.50$ & 0.47 \\
\hline Charlson score (continuous variable) & 1.10 & $1.00-1.23$ & 0.046 & 1.05 & $0.93-1.18$ & 0.20 & 1.02 & $0.91-1.16$ & 0.63 \\
\hline Surgical indication & & & 0.05 & & & 0.05 & & & 0.24 \\
\hline Relative versus Elective & 0.54 & $0.32-1.23$ & 0.11 & 0.56 & $0.31-1.00$ & 0.14 & 0.64 & $0.35-1.16$ & 0.14 \\
\hline Imperative versus Elective & 2.10 & $1.02-4.39$ & 0.03 & 1.96 & $0.95-3.94$ & 0.07 & 2.10 & $1.01-4.37$ & 0.04 \\
\hline Clinical Tumor stage & & & 0.07 & & & 0.17 & & & 0.13 \\
\hline cT1a versus cT2 & 2.13 & $1.49-2.84$ & 0.01 & 2.17 & $1.04-3.22$ & 0.02 & 1.94 & $1.03-2.79$ & 0.033 \\
\hline cT1bversus cT2 & 1.16 & $0.34-2.99$ & 0.54 & 1.21 & $0.68-3.16$ & 0.49 & 1.14 & $0.39-2.87$ & 0.59 \\
\hline PADUA score & 1.14 & $1.01-1.21$ & 0.04 & 1.12 & $1.00-1.26$ & 0.045 & 1.09 & $0.97-1.22$ & 0.13 \\
\hline Off-clamp versus clamp procedure & 1.32 & $0.99-1.47$ & 0.05 & 1.13 & $0.80-1.60$ & 0.47 & 1.07 & $0.75-1.52$ & 0.70 \\
\hline Low volume centre ( $\leq 60 \mathrm{PN} /$ year $)$ & 2.09 & $1.49-2.93$ & $<0.0001$ & 2.22 & $1.56-3.14$ & $<0.0001$ & 2.22 & $1.55-3.18$ & $<0.0001$ \\
\hline Surgical approach & & & 0.36 & & & 0.21 & & & 0.21 \\
\hline LPN versus OPN & 1.36 & $1.00-2.13$ & 0.05 & 1.52 & $1.01-2.27$ & 0.03 & 1.62 & $1.07-2.43$ & 0.02 \\
\hline RAPN versus OPN & 1.07 & $0.42-1.24$ & 0.23 & 1.09 & $0.74-1.63$ & 0.93 & 1.19 & $0.74-1.90$ & 0.47 \\
\hline Enucleoresection vs simple enucleation & 1.64 & $1.12-2.38$ & 0.01 & 1.34 & $0.88-2.04$ & 0.17 & 1.44 & $0.94-2.21$ & 0.09 \\
\hline Lymphovascular invasion & 2.70 & $1.53-4.76$ & 0.001 & - & - & - & 2.27 & $1.19-4.17$ & 0.01 \\
\hline Upstaging to pT3a & 2.89 & $1.85-4.51$ & $<0.0001$ & - & - & - & 2.81 & $1.72-4.59$ & $<0.0001$ \\
\hline Nucleolar grading $3-4$ vs. $1-2$ & 1.45 & $1.00-2.10$ & 0.046 & - & - & - & 1.36 & $0.90-2.03$ & 0.13 \\
\hline
\end{tabular}




\section{Nomogram for predicting positive surgical margins after partial nephrectomy}

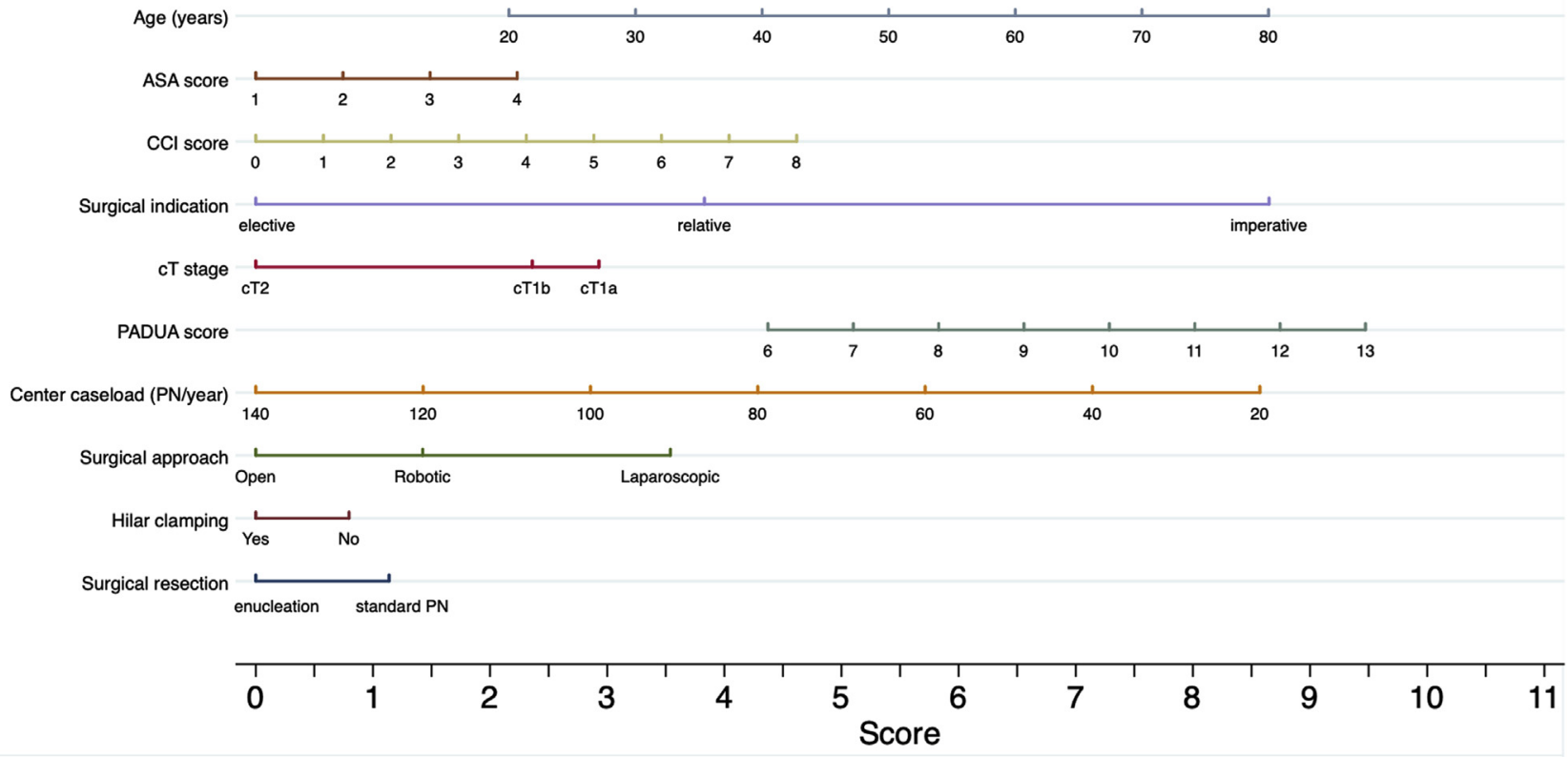

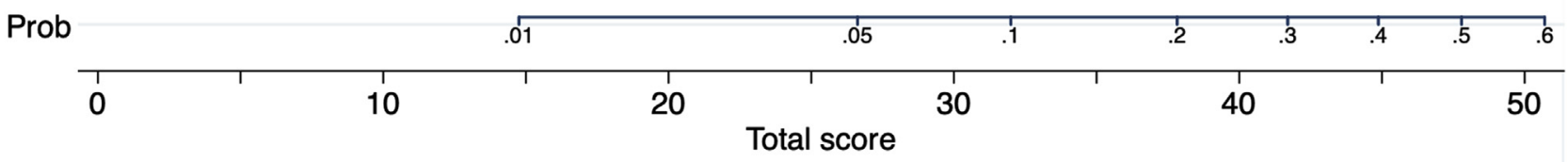

Fig. 1. Surgical nomogram for predicting positive surgical margins in patients undergoing partial nephrectomy for renal cell carcinoma (Area under the curve: 0.66 ).

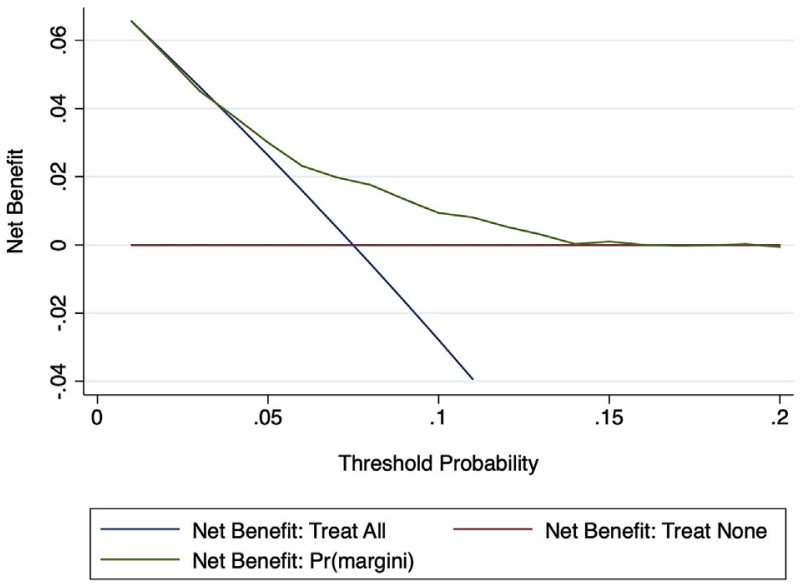

Fig. 2. Decision curve analysis for the evaluation of the clinical net benefit using the surgical nomogram for detecting positive surgical margins.

\section{Conclusions}

In this study, PSMs after PN were significantly more likely in patients with lower clinical stage, higher PADUA score, in

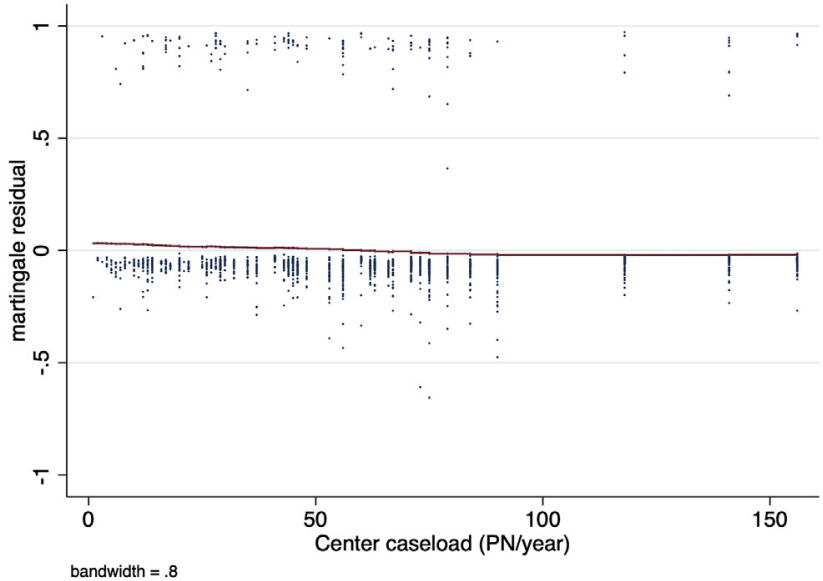

Fig. 3. Martingale residuals plot from a null multivariable model against number of partial nephrectomies per year.

individuals referred to laparoscopic PN and in those treated at lower volume centers. Notably, patients treated in centers performing $\leq 60 \mathrm{PN} /$ year have a double of risk of PSMs. Relying on such observations, for the first time, a surgical nomogram was 
constructed to predict the likelihood of PSMs in patients with localized renal tumors after PN to better drive clinical decision making.

\section{References}

[1] Albiges L, Abu-ghanem Y, Bensalah K, Giles RH, Hofmann F, Dabestani S, et al European association of Urology guidelines on renal cell Carcinoma : the 2019 update. 2019. https://doi.org/10.1016/j.eururo.2019.02.011.

[2] Campbell SC, Novick AC, Belldegrun A, Blute ML, Chow GK, Derweesh IH, et al. Guideline for management of the clinical T1 renal mass. J Urol 2009;182: 1271-9. https://doi.org/10.1016/j.juro.2009.07.004.

[3] Mari A, Di Maida F, Tellini R, Campi R, Sforza S, Cocci A, et al. Oncologic outcomes in patients treated with endoscopic robot assisted simple enucleation (ERASE) for renal cell carcinoma: results from a tertiary referral center. Eur J Surg Oncol 2019;45:1977-82. https://doi.org/10.1016 j.ejso.2019.03.045

[4] Marszalek M, Carini M, Chlosta P, Jeschke K, Kirkali Z, Knuchel R, et al. Positive surgical margins after nephron-sparing surgery. Eur Urol 2012;61:757-63. https://doi.org/10.1016/j.eururo.2011.11.028.

[5] Steinestel J, Steffens S, Steinestel K, Schrader AJ. Positive surgical margins in nephron-sparing surgery: risk factors and therapeutic consequences. World J Surg Oncol 2014;12:252. https://doi.org/10.1186/1477-7819-12-252.

[6] Borghesi M, Brunocilla E, Schiavina R, Martorana G. Positive surgical margins after nephron-sparing surgery for renal cell carcinoma: incidence, clinical impact, and management. Clin Genitourin Canc 2013;11:5-9. https://doi.org/ 10.1016/j.clgc.2012.09.010.

[7] Tellini R, Antonelli A, Tardanico R, Fisogni S, Veccia A, Furlan MC, et al. Positive surgical margins predict progression-free survival after nephron-sparing surgery for renal cell carcinoma: results from a single center cohort of 459 cases with a minimum follow-up of 5 years. Clin Genitourin Canc 2019;17: e26-31. https://doi.org/10.1016/j.clgc.2018.08.004.

[8] Kryvenko ON. Positive surgical margins increase risk of recurrence after partial nephrectomy for high risk renal tumors. Shah $\mathrm{PH}$, Moreira DM, Okhunov Z, Patel VR, Chopra S, Razmaria AA, Alom M, George AK, Yaskiv O, Schwartz MJ, Desai M, Vira MA, Richstone L, Landman J. Urol Oncol 2017;35: 449-50. https://doi.org/10.1016/j.urolonc.2017.03.013.

[9] Laganosky DD, Filson CP, Master VA. Surgical margins in nephron-sparing surgery for renal cell carcinoma. Curr Urol Rep 2017;18:8. https://doi.org 10.1007/s11934-017-0651-5.

[10] Bensalah K, Pantuck AJ, Rioux-Leclercq N, Thuret R, Montorsi F, Karakiewicz PI, et al. Positive surgical margin appears to have negligible impact on survival of renal cell carcinomas treated by nephron-sparing surgery. Eur Urol 2010;57:466-71. https://doi.org/10.1016/j.eururo.2009.03.048.

[11] Ani I, Finelli A, Alibhai SMH, Timilshina N, Fleshner N, Abouassaly R. Prevalence and impact on survival of positive surgical margins in partial nephrectomy for renal cell carcinoma: a population-based study. BJU Int 2013;111:E300-5. https://doi.org/10.1111/j.1464-410X.2012.11675.X.

[12] Shum CF, Bahler CD, Sundaram CP. Impact of positive surgical margins on overall survival after partial nephrectomy-A matched comparison based on the National Cancer Database. Urol Oncol 2018;36. https://doi.org/10.1016 j.urolonc.2017.11.009. 90.e15-90.e21.

[13] Maurice MJ, Zhu H, Kim SP, Abouassaly R. Reexamining the association between positive surgical margins and survival after partial nephrectomy in large American cohort. J Endourol 2016;30:698-703. https://doi.org/10.1089/ end.2016.0031.

[14] Ficarra V, Novara G, Secco S, Macchi V, Porzionato A, De Caro R, et al. Preoperative aspects and dimensions used for an anatomical (PADUA) classification of renal tumours in patients who are candidates for nephron-sparing surgery. Eur Urol 2009;56:786-93. https://doi.org/10.1016/ j.eururo.2009.07.040.

[15] Wood EL, Adibi M, Qiao W, Brandt J, Zhang M, Tamboli P, et al. Local tumor bed recurrence following partial nephrectomy in patients with small renal masses. J Urol 2018;199:393-400. https://doi.org/10.1016/j.juro.2017.09.072.

[16] Hu X, Shao YX, Wang Y, Yang ZQ, Yang WX, Li X. Partial nephrectomy versus ablative therapies for cT1a renal masses: a Systematic Review and metaanalysis. Eur J Surg Oncol 2019. https://doi.org/10.1016/j.ejso.2019.05.010.

[17] Minervini A, Campi R, Di Maida F, Mari A, Montagnani I, Tellini R, et al. Tumorparenchyma interface and long-term oncologic outcomes after robotic tumor enucleation for sporadic renal cell carcinoma. Urol Oncol 2018;36. https:// doi.org/10.1016/j.urolonc.2018.08.014. 527.e1-527.e11.

[18] Minervini A, di Cristofano C, Lapini A, Marchi M, Lanzi F, Giubilei G, et al. Histopathologic analysis of peritumoral pseudocapsule and surgical margin status after tumor enucleation for renal cell carcinoma. Eur Urol 2009;55: 1410-8. https://doi.org/10.1016/j.eururo.2008.07.038.

[19] Yossepowitch O, Thompson RH, Leibovich BC, Eggener SE, Pettus JA, Kwon ED, et al. Positive surgical margins at partial nephrectomy: predictors and oncological outcomes. J Urol 2008;179:2158-63. https://doi.org/10.1016/ j.juro.2008.01.100.

[20] Minervini A, Campi R, Lane BR, De Cobelli O, Sanguedolce F, Hatzichristodoulou G, et al. Impact of resection technique on perioperative outcomes and surgical margins after partial nephrectomy for localized renal masses: a prospective multicenter study. J Urol 2019. https://doi.org/10.1097/ JU.0000000000000591. 101097JU0000000000000591.

[21] Xia L, Wang X, Xu T, Guzzo TJ. Systematic review and meta-analysis of comparative studies reporting perioperative outcomes of robot-assisted partial nephrectomy versus open partial nephrectomy. J Endourol 2017;31: 893-909. https://doi.org/10.1089/end.2016.0351.

[22] Bravi CA, Larcher A, Capitanio U, Mari A, Antonelli A, Artibani W, et al. Perioperative outcomes of open, laparoscopic, and robotic partial nephrectomy: a prospective multicenter observational study (the RECORd 2 project). Eur Urol Focus 2019. https://doi.org/10.1016/j.euf.2019.10.013.

[23] Mottrie A, De Naeyer G, Schatteman P, Carpentier P, Sangalli M, Ficarra V. Impact of the learning curve on perioperative outcomes in patients who underwent robotic partial nephrectomy for parenchymal renal tumours. Eur Urol 2010:58:127-32. https://doi.org/10.1016/j.eururo.2010.03.045.

[24] Dagenais J, Bertolo R, Garisto J, Maurice MJ, Mouracade P, Kara O, et al. Variability in partial nephrectomy outcomes: does your surgeon matter? Eur Urol 2019;75:628-34. https://doi.org/10.1016/j.eururo.2018.10.046.

[25] Peyronnet B, Tondut L, Bernhard J-C, Vaessen C, Doumerc N, Sebe P, et al. Impact of hospital volume and surgeon volume on robot-assisted partial nephrectomy outcomes: a multicentre study. BJU Int 2018. https://doi.org/ 10.1111/bju.14175.

[26] Couapel J-P, Bensalah K, Bernhard J-C, Pignot G, Zini L, Lang H, et al. Is there a volume-outcome relationship for partial nephrectomy? World J Urol 2014;32: 1323-9. https://doi.org/10.1007/s00345-013-1213-1.

[27] Minervini A, Campi R, Sessa F, Derweesh I, Kaouk JH, Mari A, et al. Positive surgical margins and local recurrence after simple enucleation and standard partial nephrectomy for malignant renal tumors: systematic review of the literature and meta-analysis of prevalence. Minerva Urol Nefrol 2017. https:// doi.org/10.23736/S0393-2249.17.02864-8.

[28] Mari A, Morselli S, Sessa F, Campi R, Di Maida F, Greco I, et al. Impact of the offclamp endoscopic robot-assisted simple enucleation (ERASE) of clinical T1 renal tumors on the postoperative renal function: results from a matched-pair comparison. Eur J Surg Oncol 2018. https://doi.org/10.1016/ j.ejso.2018.01.093.

[29] Bertolo R, Simone G, Garisto J, Nakhoul G, Armanyous S, Agudelo J, et al. Offclamp vs on-clamp robotic partial nephrectomy: perioperative, functional and oncological outcomes from a propensity-score matching between two highvolume centers. Eur J Surg Oncol 2019. https://doi.org/10.1016/ j.ejso.2018.12.005

[30] Cao DH, Liu LR, Fang Y, Tang P, Li T, Bai YJ, et al. Simple tumor enucleation may not decrease oncologic outcomes for T1 renal cell carcinoma: a systematic review and meta-analysis. Urol Oncol Semin Orig Investig 2017;35. https:// doi.org/10.1016/j.urolonc.2017.07.007. 661.e15-661.e21. 